\title{
Science as Representation: Flouting the Criteria
}

\author{
Bas C. van Fraassen ${ }^{\dagger}$
}

Criteria of adequacy for scientific representation of the phenomena pertain to accuracy and truth. But that representation is selective and may require distortion even in the selected parameters; this point is intimately connected with the fact that representation is intentional, and its adequacy relative to its particular purpose. Since observation and measurement are perspectival and the appearances to be saved are perspectival measurement outcomes, the question whether this "saving" is an explanatory relation provides a new focus for the realist/antirealist debate. The Born rule and von Neumann's "collapse" postulate in quantum mechanics provide a telling case for this question in recent physics.

1. Introduction. Science is a representation of nature, in mathematical form, accomplishing by this means . . . a certain end that philosophers debate. Criteria of success or completeness for scientific representation must be related to this end, but appear concretely in science itself in theory choice and evaluation. Remarkably, scientific progress at times involves precisely the rejection of previously proclaimed criteria. But the aftermath of such a rejection is quite typically a reactionary philosophical effort at restoration.

2. Three Completeness Criteria. The Aristotelian ideal that science must explain how things happen by demonstrating that they must happen in the way they do was rejected in the time of Galileo, Gassendi, Boyle, Descartes, and Newton. But the reaction set in with their own and other writings of the period as they focus on the idea of laws of nature - and both Leibniz and Descartes even suggested an a priori foundation for these laws.

Modern science recognized determinism as a new criterion: a scientific account of a phenomenon is not complete until it represents this phe-

$\dagger$ To contact the author, please write to: Philosophy Department, Princeton University, Princeton, NJ 08544-1006; e-mail: fraassen@princeton.edu.

Philosophy of Science, 71 (December 2004) pp. 794-804. 0031-8248/2004/7105-0012\$10.00 Copyright 2004 by the Philosophy of Science Association. All rights reserved. 
nomenon as part of a deterministic process. Supporting this criterion, or perhaps deriving from its force, was the philosophical creed that the very intelligibility of nature and the very coherence of experience require their possibility of being conceivable as set in a rigidly deterministic causal order.

This criterion was vocally rejected by quantum physicists, and the success of the new quantum theory was attributed in part to its resolute acceptance of an irreducible indeterminism in nature. With one criterion gone, another had to be put in place, and Reichenbach offered an apparently weaker but still substantive new completeness criterion: the Common Cause Principle. ${ }^{1}$ This principle is satisfied by the causal models in general use in the social sciences and for many purposes in the natural sciences as well. But the violation of Bell's inequalities shows that even this third criterion was rejected, in effect, by the new physics. Here too, as we well know, there has been a good deal of reactionary efforts at restoration, the most successful being Bohm's mechanics-which surprisingly presents a picture of 'determinism without causality' so to speak.

3. Appearance and Reality. There was a fourth completeness criterion much more deeply ingrained in modern physics; but that too was one clearly, emphatically, and explicitly rejected in the Copenhagen school. Physics in the modern era depicted reality as quite different from the appearances, but accepted the criterion that a complete physics must explain how those appearances are produced in reality. The disparity was salient when Galileo and Gassendi embraced atomism: those atoms have only primary properties while the appearances are colorful, noisy, smelly, and tasty. Descartes, though not an atomist, went further by restricting the real attributes of matter to extension in space and time. Newton's forces and masses certainly don't diminish this disparity, nor do the classical fields whether adding to or replacing matter in this representation of the real physical world.

What does "explain" mean in this context? Each of the three criteria I gave as examples before this one involve modality (whether physical, metaphysical, or logical necessity). So does the fourth, which I'll call the appearance-from-reality criterion, for it is a demand for explanation, satisfiable only by connections deeper than brute or factual regularity. We credit modern science with adequate and satisfactory explanations of how many familiar phenomena are produced: how ash is produced when we

1. See van Fraassen 1982; under certain conditions this criterion actually demands determinism. But from the example of Bohmian mechanics we can also see that satisfaction of this criterion is not logically implied by determinism; see further notes below. 
burn a cigarette or some logs, how methane is naturally produced in a swamp, and how a flame is turned yellow when a sodium sample is inserted. These effects are not simply predicted to occur under suitable conditions: we are shown how they are produced in nature. The last example is already one in which an aspect of appearance (color) is explained, and this explanation is continued from optics to the physiology of vision. In this way the scientific representation of nature is shown to include the appearances in question not just as fitting into that representation (that would be a minimal requirement - the one Bellarmini suggested to Galileo as solely relevant), but as produced as proper part of the reality depicted.

Before turning to the rejection of this criterion in the twentieth century, I want to explain the form its satisfaction took in modern physics, for the most basic attributes of the appearances, namely, their spatial and temporal form. Recent philosophy of mind contains arguments against the physicalist reducibility of such properties as color, but however that may be (cf. Giere 2000), the rejection I am talking about goes much deeper.

4. Phenomena vs. Visual Appearances. There were two developments in techniques of representation before Galileo that we can see as feeding into the kinematic representation developed in his century. The first was that of linear one-point perspective in painting, and the second Copernicus' and Tycho's mastery of transforming geometric models in astronomy so as to shift the center taken as 'at rest'. Both concentrated on how a description of the visual appearance from particular vantage points can be derived from a reality admitting of many different vantage points. Both grew from the subject of Perspectiva, a melange of geometry, optics, and practical drafting techniques, and both were steps on the way to projective and descriptive geometry. But the sorts of representation they provided were more than superficially different.

Linear perspective in painting began with Giotto in the fourteenth century. Theory was not far behind: early in the fifteenth century Brunelleschi's "experiment" demonstrated the perspectival technique, and Vasari's monograph proclaimed that the new art of "painting is nothing more than the simple portrayal of all things alive in nature by means of design and color as nature herself produces them" (cited Gombrich 1960, 12). But that this was in effect a method of measurement, with the drawing as measurement outcome, was clearly recognized upon more mathematically oriented reflection. Its techniques derived from the geometry of Euclid's Optics, gave birth to the early stages of projective geometry (Pascal, Desarques), and were at least theoretically mechanized in Dürer's revealingly titled Art of Measurement.

The content of such a visual perspective is precisely the content of a 
complex, technically advanced measurement outcome. For every triple consisting of a point in space, an orientation, and a plane cutting that orientation, there is a one-point linear perspective projecting the world onto that plane. We should recognize this as a crucial general observation concerning all measurement: measurement is perspectival. The content of any measurement outcome, whether a proposition or a diagram, is indexical: it is not how things are but how they look "from here."

Representations of the solar system by Copernicus and Tycho were such that each could refer to the other as showing the way things look from a different vantage point. Yet we must be clear that they were not constructing content of visual perspectives in the way of the painter Vasari. The latter constructed, as it were, the outcome of a single measurement. The astronomical models were three dimensional, and what was shown in them was what the content had to be from any chosen vantage point. It would indeed have been natural for Copernicus to say that Tycho's or Ptolemy's model just showed the content of the earth-bound perspective. Natural, but inaccurate: in the same way that it is natural but inaccurate to speak of frames of reference (in the modern physics about to develop) as perspectival. The kinematic quantities do vary from frame to frame, but unlike the content of a visual perspective, the content of a frame does not suffer from occlusion and marginal distortion. ${ }^{2}$

Copernicus' model represents the observable phenomena, that is, certain processes in space and time. What the Copernican does in order to credential his representation is to explain by means of geometric optics and projective geometry how the visual appearances (content of outcomes of measurements made by astronomers) are produced from his reality. When frames of reference came into their own, we had eventually a three-level representation: there is the world as described in coordinate independent terms, then the world as described in a given frame of reference (coordinatization), and finally the world as it looks from a given vantage point with specific orientation. The first admits of many of the second sort, and the second of many of the third sort. In the modern era, each level has a certain completeness, in that the higher level is uniquely determined by the collection of those at the next level plus the transformations that connect them - but also a representation on the first or second level 'contains everything' in a way that the third most definitely cannot. ${ }^{3}$ With special relativity, all this can still be said; with general relativity we must

2. Such limiting features may be crucial to the distinction between visual picturing and other modes of representation; see Lopes 1996, ch. 6.

3. We may reasonably suspect that the conviction that this is so helped to inspire the "construction" programs of Russell's Our Knowledge of the External World and Carnap's Aufbau. 
already admit that a single frame of reference, like a two-dimensional map of any part of the Earth, cannot 'contain everything' in that sense. But there is no diminishing of the rightful claim that both the phenomena and the kinematic appearances from any given vantage point in space and time can be shown to be produced within the depicted reality.

5. The Strange Case of Quantum Mechanics. The vehicle for prediction in quantum mechanics is, at heart, the Born Rule: If observable $A$ is measured on a system in quantum state $\psi$, the expectation value of the outcome is $\langle\psi, A \psi\rangle$. Measurement outcomes are the appearances; the quantum states are the theoretically described reality. For the Born rule to yield checkable predictions of the appearances to us we must be willing to lay down at least as hypotheses that a given recognizable object has a certain quantum state and that a certain recognizable process is an $A$-measurement. The famous measurement problem is that our two descriptions, one of what happens at the level we can observe and one of the processes as modeled in quantum mechanics, do not match.

As far as prediction goes, the riches gained have been beyond the dreams of avarice - but no explanation of how the observed phenomena derive from the quantum state via the character of object and measurement setup has been forthcoming. This 'gap' in our understanding of nature (as it was indeed described) has engendered a plethora of attempted reinterpretations of the quantum theory, in opposition to the Copenhagen creed that there really is no gap to be filled.

How much does the Born rule say? If different measurement outcomes are compatible with the simultaneous quantum state of the whole setup (object plus apparatus plus any part of the environment from which that is not isolated) then the measurement outcomes do not supervene on the theoretically described reality. But that "if" does not follow from what I have said so far. The Born rule starts with the initial quantum state of the object; it does not rule out that the measurement outcome supervenes on or even derives from the final state of the object, or of the object + apparatus. But is that so? Can it be so?

Heisenberg was the most straightforward advocate for the view that the Born rule is enough and completes the task of physics. In contrast, von Neumann and Wigner provided the most salient example of wishing to add to the theory so as to satisfy the appearance-from-reality criterion (see below). ${ }^{4}$ Since then it seems to me that the currently more or less acceptable interpretations offered fall into three classes: (i) the sort that

4. There have of course been others, such as Prugovecki's nonlinear corrections to the Schrödinger equation and the GRW indeterministic 'swerve', a random partial collapse with measurable frequency. 
purport to derive the appearance from the reality but fail, and (ii) those that do not purport to do this, but either (ii-1) pay lip service to the old ideal or (ii-2) more honestly content themselves with fleshing out the Born interpretation in a way that precludes this third sort of completeness altogether. ${ }^{5}$ If that is correct, of course, then the Copenhagen school was right to reject the appearance-from-reality criterion as imperative for the sciences.

5.1. Is There a 'Collapse'? Von Neumann's projection postulate implies that in a measurement, the quantum state of the object is projected or 'collapsed' into one of the eigenstates of the measured quantity. The immediate questions are: What constitutes a measurement? and What explains this collapse? The two sorts of responses which attempted to maintain von Neumann's proposal were initially typified by Wigner on the one hand, and by Groenewold and Margenau on the other.

Wigner answered that a measurement is not an event completely describable in physics; it must include consciousness, a mind-body interaction. ${ }^{6}$ This only looks like it answers the second question, for in fact it provides no clue at all to how the appearances thus derive from the reality.

Groenewold and Margenau argued instead that von Neumann's added postulate is purely interpretative and does not really augment the Born Rule (see van Fraassen 1991, 251-252). We can illustrate their argument with Schrödinger's famous cat: the probability that at the end we will find the cat still alive is the same on both calculations, assuming that there is, or there is not, a collapse of the wave function at the midway time when the infernal device functions. The ostensibly correct conclusion is that von Neumann's postulate does not affect the empirical content of the theory. That is not correct, since there is a definable quantity pertaining

5. I will actually only look at some sorts of interpretations, and realize that both the range I inspect and my assessment of what are currently more or less acceptable interpretations are controvertible. With respect to the Bohmian option I'll again avoid a direct confrontation, but I place it in the first class.

6. Imagine Schrödinger's dismay - he wrote "For it must have given to de Broglie the same shock and disappointment as it gave to me, when we learnt that a sort of transcendental, almost psychical interpretation of the wave phenomenon had been put forward, which was very soon hailed by the majority of leading theorists as the only one reconcilable with experiments, and which has now become the orthodox creed, accepted by almost everybody, with a few notable exceptions" (Schrödinger 1953, 16). 
to the system as a whole (box with cat etc. inside) for which measurement outcome probabilities are certainly different on the two scenarios. ${ }^{7}$

Let's admit that von Neumann's alteration of the quantum theory, with or without Wigner's addition, implies that the phenomena do derive from the quantum-mechanically described reality. But the appearance-fromreality criterion is nevertheless not satisfied because physics cannot provide the derivation. The story here is that it is after all a stochastic process on the level of the quantum states themselves: they develop deterministically except for abrupt 'swerves' during a class of special interactions, the measurements. ${ }^{8}$ Very well, but then we run up against the question, "When is a measurement made?" If we answer this with the quantum theory of measurement, the final transition ends up somewhere beyond description-for there is no such discontinuous transition in an isolated system, and the setup is certainly part of some isolated system. But if we answer it with a non-quantum-mechanical description of measurement setups (as Bohr suggested we must) then we also close the door to a derivation of the outcome from the quantum mechanical process. ${ }^{9}$

These early discussions are illuminating not only because they began to chart our range of options, but also because they were closely related to practice. Whatever the theoretical status of 'collapse', the way the working physicist calculates does always assume that the appearances will be at least as if states and thus collapse in measurement. Generations of students have applied the Born Rule to solve textbook problems, imaging that quantum systems lurch from one pure state to another, simply asserting that upon measurement the object will be in one of the eigenstates of the measured observable, with given probability. The appearances are as if von Neumann's projection postulate is true.

7. Today 'recombination' experiments furnish the most psychologically compelling support for rejecting collapse, but in my view Albert's point is the most solid reason. Albert's point does not give a reason to reject collapse theories - there is no a priori reason to expect the predictions of the no-collapse theory to be vindicated, as opposed to those of a collapse theory. His point serves only to reject the Groenewold-Margenau contention that the collapse adds no empirical import.

8. That the problem I am raising does not simply derive from the indeterminism is clear because the GRW theory is not subject to the same objection. They answer the "when" question acceptably by saying that the localization is random. There is no special class of situations that are the occasions for that effect.

9. This dilemma is arguably the reason that some physicists have insisted strongly that quantum mechanics is only a theory of measured systems. This sort of view is to be contrasted with one congenial especially to cosmologists, and I think most discussants from the side of philosophy, to the effect we are to think of quantum mechanics as potentially applying as well to the universe as a whole. The choice between these two views is clearly and explicitly laid out by Wheeler's commentary (1957) on Everett's original paper. 
5.2. The Appearances Yoked to a Forbearing Reality. Jeffrey Bub's Interpreting the Quantum World displays a large class of interpretations: modal interpretations in a general sense. On all of them a physical quantity can have a determinate value even if the quantum state does not make it so (Bub 1997, 178). ${ }^{10}$ Those definite values are encoded in a second state, the 'value state' or 'property state'. No collapse is needed for measurement outcomes: while in a quantum state which does not imply that at all, the object is as if it is an eigenstate of the pertinent observable.

I propose a new way to understand such interpretations. Do not take them as attributing a dual-aspect character to reality, but rather as representing reality (the quantum states) and appearance (the value states) jointly but separately. The value states are then to be understood as containing the contents of possible measurement outcomes, these contents being representable as indexical propositions (describing, in effect, how things look in a possible but specific measurement setup). See how this is at the same time like and different from how Copernicus accompanied his sun-centered model with a representation of how the planetary motions look from an earthly vantage point. The difference is only that in Copernicus' case, the latter was derivable and explainable on the basis of the former.

In the case of visual perspectives as treated in projective geometry, we also think of every point and orientation determining a perspective, regardless of whether there is a thus-oriented measurement apparatus or viewer present at that point. Think of it here in the same way. The appearances are the contents of possible as well as actual measurement outcomes. ${ }^{11}$

Thus the appearances are saved - but in a way that explicitly violates the appearance-from-reality criterion. The appearances do not even su-

10. This class of interpretations include Bohm's interpretation, Bub's own, versions of Bohr, Kochen, and many others, though it does not in fact include all modal interpretations - see further note below. The Copenhagen Variant of the Modal Interpretation, which I shall discuss below, is not included, but shares the features I am outlining here.

11. This must be read very carefully. All those measurement outcome contents must cohere together in a certain way, so that they can be thought of as all perspectives on a single world in some specific quantum state. In just the same way, the entire set of contents of visual perspectives, with origins in both possible and actual viewers, in a given room for example, must cohere so that they can be regarded as being "of" the same room. In the case of the modal interpretations I am discussing, the delineation of what the joint value states can be of the parts of a compound system, given a quantum state for the whole, is directed to this point. For an application to sequential measurement, see van Fraassen 1997. 
pervene on the reality, for two systems in the same quantum state may have different value states.

6. The Structure of Appearance. What are the appearances like, on these interpretations? We do see quite some variation there. ${ }^{12}$ Bub's interpretation implies that the actual state of the world is characterized by the definiteness of a single 'privileged' observable. That quantity need not be position. We can think of his world as follows: it has a quantum state, and in addition to that there is an observable which has a definite value, just as if that observable was just measured on the world, with a collapse precipitated by that measurement. Note well that this is a matter of appearance only: the quantum state is not collapsed.

My own favorite interpretation, the Copenhagen Variant of the Modal Interpretation (CVMI), relates to these more or less as windowing does to one-point perspective. ${ }^{13}$ "Windowing" is the artist David Hockney's term for a perspective-violating technique in painting, amply evident both in the Renaissance and in the modern era well before Cézanne's more drastic departures (Hockney 2001, 99-121). There is in such paintings no single privileged vantage point but a number of them, to which the eye (or the imagination) adjusts smoothly as it moves around the scene. Similarly in the Copenhagen Variant of the Modal Interpretation there is no simple privileged observable. But it is as if the Ignorance Interpretation of Mixtures is correct, for every object in the world has a 'value state' that is pure. These value states are related to the quantum states and to measurement processes (quantum mechanically defined) so that in consequence it is also as if the projection postulate is true. Again the 'as if' describes the appearances, that is, the value states (which include the measurement outcomes) but not the quantum state.

Why do I say that this is like 'windowing' rather than like one-point

12. Although Bub lists it as one of the interpretations covered in his framework, I am not going to take up Bohmian mechanics here. Bohm allows only one parameter to have a definite value - always the same one, always definite - namely position. This world is one of particles that are always somewhere - and larger objects 'made up' of those particles, always in a precise spatial region. Their motions are continuous in time. This view may have been inspired by the extreme operationalist idea, going back to Mach, that in the last analysis every measurement is a length measurement. (Not very plausible: could you describe even a length measurement operation using only predicates denoting lengths?) Or perhaps it derives even further back from Descartes' dream of a world whose only objective properties are attributes of extension. That the phenomena are saved in a weak sense only and that there is still an appearance/reality gap here is argued, in effect, in van Fraassen 1994 as well as in Stone 1994 and Bedard 1999.

13. See van Fraassen 1998 for an explanation of how the Copenhagen Variant of the Modal Interpretation is related to, but does not fall in, the class described in Bub 1997. 
perspective? On the CVMI every object, including every part of an object, 'looks' as if it has just been projected into some pure state. This implies that we cannot say: it is just as if the 'collapse' idea is right and the world looks as if it has just been subjected to great single comprehensive measurement. For if a compound system is already in a pure state then its appearance matches its reality. But in general there is no projection of that state of the whole which also sends the components into pure states. Yet the value states are such that it is possible, e.g., for both the whole and the parts to be definite in their apparent characteristics (to the extent that compatibility of observables allows). So it is as if each is seen individually from some measuring vantage point.

7. The Final Challenge. The details of quantum-theory interpretation are fascinating, challenging, and frustrating, and its problems are by no means all settled. But my main aim in this paper is not to defend a specific interpretation-let alone its details in one form or another! Rather, what I mean to do is to argue the thesis that this actual part of recent history of science should convince us that it is perfectly scientific, and scientifically acceptable, to reject the completeness criteria for science that I outlined. Although I take it that all of the foregoing could be granted by today's scientific realist if by anyone, my thesis concerning the aim and methodology of science appears to clash with at least certain traditional themes in 'realist' philosophies of science.

If my view of it is right, and if in addition the Copenhagen physicists were acting in a way that counts as real physics when they introduced and developed quite explicitly a theory and an interpretation incompatible with the appearance-from-reality completeness criterion, then that criterion is not a constraint on the sciences. It is, in that case, just another of those philosophically or metaphysically motivated imperatives that could hamper science if they were obeyed; though they receive much lip service, they are quickly flouted when that hampering is felt.

\section{REFERENCES}

Bedard, K. (1999), “Material Objects in Bohm's Interpretation”, Philosophy of Science 66: 221-242.

Bub, Jeffrey (1997), Interpreting the Quantum World. Cambridge: Cambridge University Press.

Giere, Ronald N. (2000), "The Perspectival Nature of Scientific Observation", paper delivered at PSA 2000, November 4, 2000. Vancouver, British Columbia.

Gombrich, Ernst Hans (1960), Art and Illusion: A Study in the Psychology of Pictorial Representation. New York: Pantheon Books.

Hockney, David (2001), Secret Knowledge: Rediscovering the Lost Techniques of the Old Masters. New York: Penguin Putnam.

Lopes, Dominic (1996), Understanding Pictures. Oxford: Oxford University Press. 
Schrödinger, Erwin (1953), "The Meaning of Wave Mechanics", in A. George, Louis de Broglie: Physicien et Penseur. Paris: Editions Albin Michel, 16-30.

Stone, A. (1994), "Does the Bohm Theory Solve the Measurement Problem?" Philosophy of Science 61: 250-256.

van Fraassen, Bas C. (1982), "Rational Belief and the Common Cause Principle", in Robert McLaughlin (ed.), What? Where? When? Why? Essays in Honor of Wesley Salmon. Dordrecht: Reidel, 193-209.

(1991), Quantum Mechanics: An Empiricist View. Oxford: Oxford University Press. - (1994), "Interpretation of QM: Parallels and Choices", in Luigi Accardi (ed.), The Interpretation of Quantum Theory: Where Do We Stand? Rome: Istituto della Enciclopedia Italiana (distributed by Fordham University Press), 7-14.

(1997), "Modal Interpretation of Repeated Measurement: Reply to Leeds and Healey", Philosophy of Science 64: 669-676.

(1998), review of Bub 1997, Foundations of Physics 28: 683-689.

Wheeler, J. (1957), “Assessment of Everett's 'Relative State' Formulation of Quantum Theory”, Reviews of Modern Physics 29: 463-465. 